\title{
Optimal Variable Sample Size and Sampling Interval Control Chart for the Process Mean based on Expected Average Time to Signal
}

\author{
Khai Wah Khaw, XinYing Chew, Sin Yin Teh, and Wai Chung Yeong
}

\begin{abstract}
A control chart is one of the effective tools in statistical process control (SPC) for improving productivity, reducing defective products and providing diagnostic information. Control charting techniques have gained increasing importance recently due to the rapid advancement in technology. Many industries tend to use control charts to monitor the quality of their products or services. The adoption of variable sample size and sampling interval (VSSI) strategy significantly improved the sensitivity of Shewhart $\bar{X}$ chart in detecting small and moderate process mean shifts, in terms of average time to signal (ATS) criterion when the process shifts are specified. However, for some scenarios in real industries, the process shift size is not set to a specific value. In this case, the expected average time to signal (EATS) can be used as a measure of performance when the process shift is unknown. The EATS of the optimal VSSI $\bar{X}$ chart is numerically evaluated based on a Markov chain approach. The findings show that the VSSI $\bar{X}$ chart prevails over the Shewhart $\bar{X}$ chart under comparison. Being able to vary the sample size and sampling interval, a practitioner will have more flexibility and better control of the process and at the same time is able to detect an out-of-control signal quicker.
\end{abstract}

Index Terms-Expected average time to signal, Markov chain, process mean, variable sample size and sampling interval.

\section{INTRODUCTION}

The continuous development of SPC is driven for on-line quality control in mass production systems. It improves the quality of products and increases profits, as well as reduces errors and rework in a process. Dr. Shewhart presented the first control chart as $\bar{X}$ chart in 1924. The applications of control charts are widely implemented in real industries. This can be shown through a wide variety of recent research publications (see [1]-[4], to name a few). Reference [5] illustrated the use of control charts in the manufacturing industry, to a product of Swat Pharmaceutical Company. Control charts were used to analyze, control and improve accounting processes [6]. For example, it could be used for detecting the errors in payroll function and tax preparation. Reference [7] applied the control charts in dairy herd

Manuscript received August 15, 2019; revised October 5, 2019.

Khaw Wah Khaw and Sin Yin Teh are with the School of Management, Universiti Sains Malaysia, 11800 Pulau Pinang, Malaysia (e-mail: khaiwah@usm.my, tehsyin@usm.my).

XinYing Chew is with the School of Computer Sciences, Universiti Sains Malaysia, 11800 Pulau Pinang, Malaysia (e-mail: xinying@usm.my).

Wai Chung Yeong is with the School of Mathematical Sciences, Sunway University, Petalling Jaya, Malaysia (e-mail: waichungy@sunway.edu.my). management. Additionally, the control chart is commonly applied to monitor the manufacturing process [8].

The Shewhart $\bar{X}$ chart is not sensitive in detecting small and moderate process shifts. To circumvent this problem, adaptive strategies were adopted into the Shewhart $\bar{X}$ chart. A control chart is considered adaptive when at least one of its designed parameters varies as a function of process data [9]. Reference [10] revealed that the designs of adaptive types chart are extensively explored by researchers. Numerous research showed that adaptive strategies significantly improved the capability of control charts in detecting small and moderate process shifts [11]-[13].

The first adaptive chart was proposed by varying the sampling interval, namely variable sampling interval (VSI) $\bar{X}$ chart [14]. Another set of studies focus on adapting the sample size (VSS) was investigated by [15] and [16] independently. Subsequently, both the parameters for sample size and sampling interval (VSSI) were made variable [17]. Reference [18] introduced a variable parameter (VP) $\bar{X}$ chart by varying the sample size, sampling interval and constant width.

A VSSI scheme is one of the adaptive control charting methods. It is well known that the VSSI scheme has been proven to be practically useful in industries compared to non-adaptive counterparts. The VSSI scheme is a practical and flexible approach in increasing the effectiveness of the Shewhart $\bar{X}$ chart, for detecting small and moderate mean shifts. The $\bar{X}$ chart provides the flexibility to quality practitioners for varying the sample size and sampling interval.

Consequently, research involving VSSI control charts continue to generate interest among researchers over the years and the VSSI scheme have been incorporated into a wide variety of different types of control charts. For example, an optimal VSSI mean squared error control chart was investigated to monitor the process mean and variance [19]. Reference [20] suggested a special VSSI chart for the process mean, with two variable sampling intervals, three sample sizes, two sets of warning limits, and a set of control limit. The optimal VSSI $\bar{X}$ chart was also proposed by using the estimated parameters [21] and adjusted loss function [22]. Reference [23] introduced the VSSI median control chart with estimated parameters and measurement errors while reference [24] studied the effect of measurement errors on the performance of the VSSI $\bar{X}$ chart. References [25] and [26] considered the VSSI chart for the process mean using auxiliary information and the coefficient of variation, respectively. More recently, a new VSSI risk-based $\bar{X}$ chart was presented by [27]. 
Meanwhile, the implementation of the VSSI charts for monitoring the multivariate process is proposed. Reference [28] discussed the implementation of the VSSI Hotelling's $T^{2}$ chart with the combination of three sample sizes and two sampling intervals. The VSSI multivariate coefficient of variation chart was developed to monitor certain processes which the mean and variance of the process data may not vary independently of each other [29]. After the statistical design of the VSSI Hotelling's $T^{2}$ was proposed, the proposed chart was economically designed to investigate the implementation costs of the chart [30].

The existing literature measures the performance of VSSI $\bar{X}$ chart in terms of average time to signal (ATS) criterion. Note that ATS is the average time required to detect an outof-control signal from the time of an occurrence of a process shift. Thus, for an out-of-control process, the smaller the ATS value, the better the chart is, when the in-control ATS $\left(\mathrm{ATS}_{0}\right)$ is specified at the desired value. However, in some of the real-life situations, the process shift size is not set to a specific value. This causes difficulty in measuring ATS performance. In this paper, the VSSI $\bar{X}$ chart is evaluated in terms of expected average time to signal (EATS), in monitoring the process shifts range. Section II reviews the properties of the VSSI $\bar{X}$ chart. Meanwhile, the computation of the ATS and EATS values are derived using a Markov chain approach. Section III compares the performances of the Shewhart $\bar{X}$ and VSSI $\bar{X}$ charts, in terms of ATS and EATS criteria. Conclusions are drawn in Section IV.

\section{VSSI $\bar{X}$ CHART}

The VSSI $\bar{X}$ chart varies the sample size $(n)$ and sampling interval $(t)$ parameters. The warning limits (i.e. upper warning limit, UWL and lower warning limit, LWL) and control limits (i.e. upper control limit, UCL and lower control limit, LCL) are employed to divide the VSSI chart into three regions, namely central, warning and out-ofcontrol regions. For the VSSI $\bar{X}$ chart, the sample size is varied between small $\left(n_{1}\right)$ and large $\left(n_{2}\right)$ sample size, where $n_{1}<\mathrm{ASS}_{0}<n_{2}$, while the sampling interval is varied between short $\left(t_{1}\right)$ and long $\left(t_{2}\right)$ sampling interval, where $t_{1}<\mathrm{ASI}_{0}<t_{2}$. Note that $\mathrm{ASS}_{0}$ and $\mathrm{ASI}_{0}$ are the specific values of the in-control average sample size and in-control average sampling interval, respectively. The control and warning limits of VSSI $\bar{X}$ chart are denoted as

$$
\begin{aligned}
\mathrm{UCL}_{i} & =\mu_{0}+k \sigma_{\bar{x}_{i}} \text { and } \mathrm{LCL}_{i}=\mu_{0}-k \sigma_{\bar{x}_{i}} \\
\mathrm{UWL}_{i} & =\mu_{0}+w \sigma_{\bar{x}_{i}} \text { and } \mathrm{LWL}_{i}=\mu_{0}-w \sigma_{\bar{x}_{i}}
\end{aligned}
$$

where $\mu_{0}$ is the mean of the process when the process is incontrol, $\sigma_{\bar{X}_{i}}$ is the standard deviation of the $\bar{X}$, for $i=1,2$. $k$ and $w$ are the control and warning limit coefficients, respectively, with $k>w$. In this paper, a single value of $w$ and $k$ is adopted to simplify the implementation and operation of the VSSI $\bar{X}$ chart [17].

The operation of the VSSI strategy (see Fig. 1) that adopted by [17] is implemented according to the following rules:

(i) if the $(i-1)$ th sample point falls in the central region (LWL, UWL), the process is in-control, and $n_{1}$ and $t_{2}$ should be adopted for computing the current sample point,

(ii) if the $(i-1)$ th sample point falls in the warning region ([LCL, LWL] or [UWL,UCL]), the process is in-control, and $n_{2}$ and $t_{1}$ should be adopted for computing the current sample point and

(iii) if the $(i-1)$ th sample point falls beyond the control limits $((-\infty, \mathrm{LCL})$ or $(\mathrm{UCL}, \infty))$, the process is outof-control and corrective actions should be taken immediately to identify the assignable cause(s).

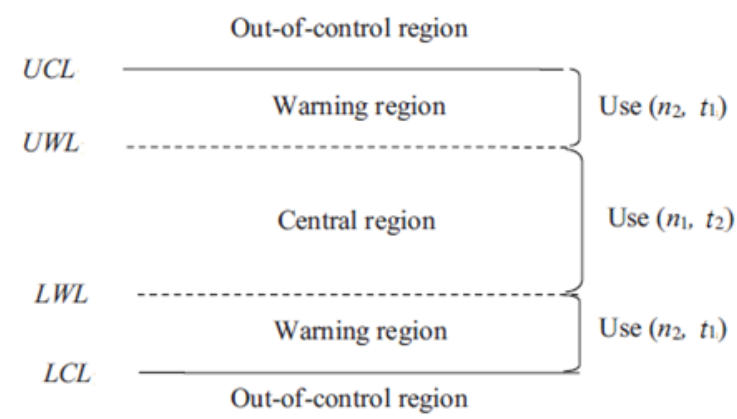

Fig. 1. Graphical view of VSSI $\bar{X}$ chart.

To ensure a fair comparison between the VSSI $\bar{X}$ and Shewhart $\bar{X}$ chart, their in-control performances must be matched. The VSSI $\bar{X}$ chart is designed so that its $\operatorname{ASS}_{0}\left(=n_{0}\right)$ and $\operatorname{ASI}_{0}\left(=t_{0}\right)$ are equal to that of $n$ and $t$ of the Shewhart $\bar{X}$ chart. The Markov chain approach is used to compute the ATS and EATS of the VSSI $\bar{X}$ chart. Three states are defined in the Markov chain model, i.e. central state, warning state and out-of-control state, which shows as follows:

State 1: $\hat{\gamma} \in[\mathrm{LWL}, \mathrm{UWL}]$

State 2: $\hat{\gamma} \in[\mathrm{LCL}, \mathrm{LWL} \cup \mathrm{UWL}, \mathrm{UCL}]$

State 3: $\hat{\gamma} \notin[\mathrm{LCL}, \mathrm{UCL}]$

The out-of-control state can be denoted as an absorbing state. The transition probability matrix with absorbing state is

$$
P=\left(\begin{array}{lll}
P_{11} & P_{12} & P_{13} \\
P_{21} & P_{22} & P_{23} \\
P_{31} & P_{32} & P_{33}
\end{array}\right)
$$

where $P_{i j}$ refers to the transition probability from the prior state $i$ to the current state $j$. Hence, the transition probabilities for the transient states in (3) are computed as

$$
\begin{gathered}
P_{11}=\operatorname{Pr}\left[|Y|<w_{1} \mid Y \sim N\left(\delta \sqrt{n_{1}}, 1\right)\right. \\
P_{12}=\operatorname{Pr}\left[w_{1}<|Y|<k_{1} \mid Y \sim N\left(\delta \sqrt{n_{1}}, 1\right)\right. \\
P_{21}=\operatorname{Pr}\left[|Y|<w_{2} \mid Y \sim N\left(\delta \sqrt{n_{2}}, 1\right)\right.
\end{gathered}
$$




$$
P_{22}=\operatorname{Pr}\left[w_{2}<|Y|<k_{2} \mid Y \sim N\left(\delta \sqrt{n_{2}}, 1\right)\right.
$$

The ATS of the VSSI $\bar{X}$ chart is obtained as

$$
\operatorname{ATS}=b^{\prime}(I-Q)^{-1} t
$$

where $\boldsymbol{b}^{\prime}=\left(b_{1}, b_{2}\right)$ is the vector of starting probabilities such that $b_{2}=1-b_{1}$ when the process is in-control. $I$ and $Q$ are $2 \times 2$ identity matrix and transition probability matrix for transient states, respectively, while $\boldsymbol{t}=\left(t_{2}, t_{1}\right)$ is the vector of the sampling interval. Subsequently, the vector of starting probabilities are given as

$$
b_{1}=\left(\frac{P_{11}^{0}}{P_{11}^{0}+P_{12}^{0}}\right)
$$

and

$$
b_{1}=\left(\frac{P_{22}^{0}}{P_{21}^{0}+P_{22}^{0}}\right)
$$

subject to $n_{0}=b_{1} n_{1}+b_{2} n_{2}$ and $t_{0}=b_{1} t_{2}+b_{2} t_{1}$.

In the computation of ATS, the process shift sizes need to be specified a priori. Hence, the EATS is vital to measure the statistical performance in situations where practitioners could not specify the process shift size. For the non-adaptive type control chart, a similar type of performance measure was employed, namely expected average run length (EARL). In this paper, the in-control EATS $\left(\right.$ EATS $_{0}$ ) is set equal to the $\mathrm{ATS}_{0}$, whereas the out-of-control EATS is obtained as

$$
\operatorname{EATS}_{1}=\int f_{\delta}(\delta) \operatorname{ATS}_{1}\left(n_{1}, n_{2}, t_{1}, t_{2}, w, k, \delta\right) d \delta
$$

where $f_{\delta}(\delta)$ refers to density probability function of $\delta$. If no information of $f_{\delta}(\delta)$ is available, it is reasonable to assume that $\delta$ follows a uniform distribution over the interval $\left(\delta_{\min }, \delta_{\max }\right)$, where $\delta_{\min }$ and $\delta_{\max }$ are defined as the minimum and maximum shift size, respectively [26].

The values of $n_{0}, t_{0}, t_{1}, k$ and $\mathrm{ATS}_{0}$ are specified. If ATS is used as a performance criterion, then specify $\delta$. Conversely, if EATS is used as a performance criterion, then specify $\delta_{\min }$ and $\delta_{\max }$. In this paper, the optimal parameter $\left(n_{1}, n_{2}, t_{2}, w\right)$ combinations that provide the smallest $\operatorname{ATS}_{1}(\delta)$ (or $\left.\operatorname{EATS}_{1}\left(\delta_{\text {min }}, \delta_{\text {max }}\right)\right)$ value are computed. The $n_{1}$ and $n_{2}$ are varied based on the constraint $1 \leq n_{1}<n_{0}<n_{2} \leq 30 \quad, \quad$ as these $\left(n_{1}, n_{2}\right)$ combinations practically cover a wide range for applications in manufacturing and industrial settings [26]. The parameters $t_{2}$ and $w$ can be obtained using formulae

$$
t_{2}=\frac{t_{0}\left(n_{2}-n_{1}\right)-t_{1}\left(n_{0}-n_{1}\right)}{n_{2}-n_{0}}
$$

and

$$
w=\Phi^{-1}\left\{\frac{2\left(t_{0}-t_{1}\right) \Phi k+t_{2}-t_{0}}{2\left(t_{2}-t_{1}\right)}\right\}, i=1,2
$$

The optimization procedure to compute the optimal parameter $\left(n_{1}, n_{2}, t_{2}, w\right)$ combinations of the VSSI $\bar{X}$ chart to minimize the $\operatorname{ATS}_{1}$ and EATS 1 values, for detecting the process mean shift, $\delta$ and shift interval $\left(\delta_{\min }, \delta_{\max }\right)$ is considered in this paper. Note that $t_{1}$ is set as 0.10 and 0.25 and the $\operatorname{ATS}_{0}=370$. The $w$ parameter is used to compute the UWL and LWL of the VSSI $\bar{X}$ chart using Equation (2). This optimization procedure is applied to minimize the

(1) $\operatorname{Min}_{\left\{n_{1}, n_{2}, t_{2}, \mathrm{w}\right\}} \operatorname{ATS}_{1}(\delta)$, subject to constraint $\operatorname{ATS}_{0}=370$, $\mathrm{ASS}_{0}=n_{0}$ and $\mathrm{ASI}_{0}=t_{0}$

(2) $\operatorname{Min}_{\left\{n_{1}, n_{2}, t_{2}, \mathrm{w}\right\}} \operatorname{EATS}_{1}\left(\delta_{\text {min }}, \delta_{\text {max }}\right)$, subject to constraint $\operatorname{EATS}_{0}=370, \operatorname{ASS}_{0}=n_{0}$ and $\mathrm{ASI}_{0}=t_{0}$

Subsequently, the optimization procedure of the VSSI $\bar{X}$ chart is given as

Step 1: Specify $n_{0}, t_{0}, t_{1}, \delta$ (for $\left.\operatorname{ATS}_{1}(\delta)\right)$ or $\left(\delta_{\text {max }}, \delta_{\text {min }}\right)$ (for $\left.\operatorname{EATS}_{1}\left(\delta_{\min }, \delta_{\max }\right)\right)$.

Step 2: Let $n_{1}=1$ and $n_{2}=n_{0}+1$.

Step 3: Compute $k$ using nonlinear equation solver, subject to constraint $\operatorname{ATS}_{0}=370$. Then compute $w$ and $t_{2}$ using Equations (12) and (13).

Step 4: Compute $\operatorname{ATS}_{1}(\delta)$ value (or $\operatorname{EATS}_{1}\left(\delta_{\text {min }}, \delta_{\text {max }}\right)$ value) using Equation (8) (or Equation (11)) with the optimal parameter combination $\left(n_{1}, n_{2}, t_{2}, w\right)$ obtained from Steps $1-3$.

Step 5: Let $n_{1}+1$ while maintaining the same value of $n_{2}$.

Step 6: Repeat Steps $3-5$ until $n_{1}=n_{0}-1$.

Step 7: Reset $n_{1}=1$ and let $n_{2}+1$.

Step 8: Repeat Steps $3-7$ until $n_{2}=30$. Here, $n_{2}=30$ can be viewed as a guideline. The practitioner will decide the maximum value of the sample size by depending on the requirement of the process.

Step 9: Select the parameter combination $\left(n_{1}, n_{2}, t_{2}, w\right)$ that minimizes the $\operatorname{ATS}_{1}(\delta)$ value (or $\operatorname{EATS}_{1}\left(\delta_{\text {min }}, \delta_{\text {max }}\right)$ value) as the optimal parameter combination.

\section{NUMERICAL COMPARISON}

The Shewhart $\bar{X}$ chart has its sampling interval set as $t=$ 1. Thus, the chart's average run length (ARL) value are similar to VSSI $\bar{X}$ chart's ATS value because ATS $=$ ARL $\times t$. Then, the initial sampling interval of the VSSI $\bar{X}$ chart should be set as unity. The $\mathrm{ATS}_{0}$ is set equal to 370. Table I shows the VSSI $\bar{X}$ chart's optimal parameters, i.e. $n_{1}, n_{2}, t_{2}$ and $w$ that minimize the $\mathrm{ATS}_{1}$ for $\delta \in\{0.20,0.40,0.80,1.20,1.60,2.00\}$, when $n_{0} \in\{3,5,7$, $10\}$ and $t_{1} \in\{0.10,0.25\}$. For example, to minimize ATS for detecting the process shift $\delta=0.80$, when $n_{0}=5$ and $t_{1}=0.25$, the optimal parameters are $\left(n_{1}, n_{2}, t_{2}, w\right)=(3,14$, $1.166,1.328)$.

Table II provides the VSSI $\bar{X}$ chart's optimal parameters, i.e. $n_{1}, n_{2}, t_{2}$ and $w$ that minimize the $\mathrm{EATS}_{1}$ for $\left(\delta_{\min }, \delta_{\max }\right) \in\{(0.30,1.00),(0.30,1.50),(0.30,2.00),(0.50$, $1.00),(0.50,1.50),(0.50,2.00)\}$, when $n_{0} \in\{3,5,7,10\}$ and 
$t_{1} \in\{0.10,0.25\}$. For example, to minimize $\mathrm{EATS}_{1}$ for detecting the process shift range $\left(\delta_{\min }, \delta_{\max }\right)=(0.30,2.00)$, when $n_{0}=3$ and $t_{1}=0.10$, the optimal parameters are $\left(n_{1}, n_{2}, t_{2}, w\right)=(1,29,1.069,1.787)$.

TABLE I: VSSI $\bar{X}$ ChaRT'S OPTIMAL PARAMETERS $\left(n_{1}, n_{2}, t_{2}, w\right)$ TO MINIMIZE ATS

\begin{tabular}{|c|c|c|}
\hline \multicolumn{3}{|c|}{$\mathrm{ATS}_{1}$} \\
\hline \multirow[b]{2}{*}{$\delta$} & \multicolumn{2}{|c|}{$t_{1}=0.10$} \\
\hline & $n_{0}=3$ & $n_{0}=5$ \\
\hline 0.20 & $1,30,1.073,1.802$ & $1,30,1.158,1.475$ \\
\hline 0.40 & $1,30,1.073,1.802$ & $1,30,1.158,1.475$ \\
\hline 0.80 & $1,10,1.283,1.215$ & $2,10,1.594,0.884$ \\
\hline 1.20 & $2,5,1.495,0.963$ & $3,6,2.980,0.429$ \\
\hline 1.60 & $2,4,1.990,0.672$ & $4,6,1.990,0.672$ \\
\hline \multirow[t]{2}{*}{2.00} & $2,4,1.990,0.672$ & $4,6,1.990,0.672$ \\
\hline & \multicolumn{2}{|c|}{$t_{1}=0.10$} \\
\hline$\delta$ & $n_{0}=7$ & $n_{0}=10$ \\
\hline 0.20 & $1,30,1.235,1.256$ & $1,30,1.405,1.011$ \\
\hline 0.40 & $1,30,1.235,1.256$ & $2,30,1.360,1.063$ \\
\hline 0.80 & $4,12,1.540,0.884$ & $6,14,1.900,0.672$ \\
\hline 1.20 & $5,8,2.800,0.429$ & $9,11,1.900,0.672$ \\
\hline 1.60 & $6,8,1.900,0.672$ & $9,11,1.900,0.672$ \\
\hline \multirow[t]{2}{*}{2.00} & $6,8,1.900,0.672$ & $9,11,1.900,0.672$ \\
\hline & \multicolumn{2}{|c|}{$t_{1}=0.25$} \\
\hline$\delta$ & $n_{0}=3$ & $n_{0}=5$ \\
\hline 0.20 & $1,30,1.056,1.802$ & $1,30,1.120,1.475$ \\
\hline 0.40 & $1,30,1.056,1.802$ & $1,30,1.120,1.475$ \\
\hline 0.80 & $1,12,1.166,1.328$ & $3,14,1.166,1.328$ \\
\hline 1.20 & $2,7,1.188,1.275$ & $3,8,1.500,0.839$ \\
\hline 1.60 & $2,5,1.375,0.963$ & $4,6,1.750,0.672$ \\
\hline \multirow[t]{2}{*}{2.00} & $2,4,1.750,0.672$ & $4,6,1.750,0.672$ \\
\hline & \multicolumn{2}{|c|}{$t_{1}=0.25$} \\
\hline$\delta$ & $n_{0}=7$ & $n_{0}=10$ \\
\hline 0.20 & $1,30,1.195,1.256$ & $1,30,1.338,1.011$ \\
\hline 0.40 & $1,30,1.195,1.256$ & $2,30,1.300,1.063$ \\
\hline 0.80 & $4,15,1.281,1.092$ & $7,18,1.281,1.092$ \\
\hline 1.20 & $5,9,1.750,0.672$ & $9,11,1.750,0.672$ \\
\hline 1.60 & $6,8,1.750,0.672$ & $9,11,1.750,0.672$ \\
\hline 2.00 & $6,8,1.750,0.672$ & $9,11,1.750,0.672$ \\
\hline
\end{tabular}

Table III presents the $\mathrm{ATS}_{1}$ values of the optimal VSSI $\bar{X}$ chart, based on the optimal parameters in Table I, for $\delta \in\{0.20,0.40,0.80,1.20,1.60,2.00\}$, when $n_{0} \in\{3,5,7$, $10\}$ and $t_{1} \in\{0.10,0.25\}$. The optimal VSSI $\bar{X}$ chart is compared with the Shewhart $\bar{X}$ chart. To ensure a fair comparison between the VSSI $\bar{X}$ and Shewhart $\bar{X}$ chart, the $n$ and $t$ of the Shewhart $\bar{X}$ chart are set equal to the $n_{0}$ and $t_{0}$ of the VSSI $\bar{X}$ chart. It is clear that the VSSI $\bar{X}$ chart generally outperforms the Shewhart $\bar{X}$ chart, for all process shifts and sample sizes. For example, from Table III, to minimize the $\mathrm{ATS}_{1}$ for detecting the process shift $\delta=0.20$, when $n_{0}=5$ and $t_{1}=0.25, \operatorname{ATS}_{1}=124.43$ and 177.73 for the VSSI $\bar{X}$ and Shewhart $\bar{X}$ charts, respectively, where the $\mathrm{ATS}_{1}$ value is reduced by $30 \%$ for the implementation of the VSSI $\bar{X}$ chart.

Table IV presents the ATS $_{1}$ values of the optimal VSSI $\bar{X}$ chart, based on the optimal parameters in Table II, for $\left(\delta_{\min }, \delta_{\max }\right) \in\{(0.30,1.00),(0.30,1.50),(0.30,2.00),(0.50$, $1.00),(0.50,1.50),(0.50,2.00)\}$, when $n_{0} \in\{3,5,7,10\}$ and $t_{1} \in\{0.10,0.25\}$. In terms of the EATS $_{1}$ criterion, the VSSI $\bar{X}$ chart generally prevails over the Shewhart $\bar{X}$ chart for all process shift intervals considered. For example, when $\left(\delta_{\min }, \delta_{\max }\right)=(0.50,2.00), n_{0}=3$ and $t_{1}=0.10, \mathrm{EATS}_{1}=$ 3.45 and 11.31 for the VSSI $\bar{X}$ and Shewhart $\bar{X}$ charts, respectively, where it is obvious that the VSSI $\bar{X}$ chart has the smallest EATS $_{1}$ value. In real-life situations, the practitioners rarely know the exact process shift size in the process monitoring. Based on the results in Table II, the VSSI $\bar{X}$ chart is a better choice for detecting process shift if the exact shift size cannot be specified.

TABLE II: VSSI $\bar{X}$ CHART's OPTIMAL PARAMETERS $\left(n_{1}, n_{2}, t_{2}, w\right)$ TO MiNiMIZE EATS,

\begin{tabular}{|c|c|c|}
\hline \multicolumn{3}{|c|}{ EATS $_{1}$} \\
\hline \multirow[b]{2}{*}{$\left(\delta_{\min }, \delta_{\max }\right)$} & \multicolumn{2}{|c|}{$t_{1}=0.10$} \\
\hline & $n_{0}=3$ & $n_{0}=5$ \\
\hline$(0.30,1.00)$ & $1,30,1.066,1.802$ & $2,30,1.108,1.600$ \\
\hline$(0.30,1.50)$ & $1,30,1.066,1.802$ & $2,30,1.108,1.600$ \\
\hline$(0.30,2.00)$ & $1,29,1.069,1.787$ & $2,30,1.108,1.600$ \\
\hline$(0.50,1.00)$ & $1,17,1.129,1.525$ & $2,17,1.225,1.275$ \\
\hline$(0.50,1.50)$ & $1,14,1.163,1.418$ & $2,15,1.270,1.193$ \\
\hline$(0.50,2.00)$ & $1,13,1.180,1.376$ & $3,16,1.164,1.418$ \\
\hline \multirow[b]{2}{*}{$\left(\delta_{\min }, \delta_{\max }\right)$} & \multicolumn{2}{|c|}{$t_{1}=0.10$} \\
\hline & $n_{0}=7$ & $n_{0}=10$ \\
\hline$(0.30,1.00)$ & $2,30,1.196,1.338$ & $4,30,1.270,1.193$ \\
\hline$(0.30,1.50)$ & $3,30,1.156,1.438$ & $4,30,1.270,1.193$ \\
\hline$(0.30,2.00)$ & $3,30,1.156,1.438$ & $5,30,1.225,1.275$ \\
\hline$(0.50,1.00)$ & $3,18,1.327,1.106$ & $6,20,1.360,1.063$ \\
\hline$(0.50,1.50)$ & $4,17,1.270,1.193$ & $6,19,1.400,1.016$ \\
\hline$(0.50,2.00)$ & $4,17,1.270,1.193$ & $6,18,1.450,0.964$ \\
\hline \multirow[b]{2}{*}{$\left(\delta_{\min }, \delta_{\max }\right)$} & \multicolumn{2}{|c|}{$t_{1}=0.25$} \\
\hline & $n_{0}=3$ & $n_{0}=5$ \\
\hline$(0.30,1.00)$ & $1,30,1.056,1.802$ & $1,30,1.120,1.475$ \\
\hline$(0.30,1.50)$ & $1,30,1.056,1.802$ & $2,30,1.090,1.600$ \\
\hline$(0.30,2.00)$ & $1,29,1.058,1.787$ & $2,30,1.090,1.600$ \\
\hline$(0.50,1.00)$ & $1,17,1.107,1.525$ & $2,19,1.161,1.345$ \\
\hline$(0.50,1.50)$ & $1,15,1.125,1.457$ & $3,18,1.115,1.492$ \\
\hline$(0.50,2.00)$ & $1,14,1.136,1.418$ & $3,18,1.115,1.492$ \\
\hline \multirow[b]{2}{*}{$\left(\delta_{\min }, \delta_{\max }\right)$} & \multicolumn{2}{|c|}{$t_{1}=0.25$} \\
\hline & $n_{0}=7$ & $n_{0}=10$ \\
\hline$(0.30,1.00)$ & $2,30,1.163,1.338$ & $4,30,1.225,1.193$ \\
\hline$(0.30,1.50)$ & $3,30,1.130,1.438$ & $4,30,1.225,1.193$ \\
\hline$(0.30,2.00)$ & $3,30,1.130,1.438$ & $5,30,1.188,1.275$ \\
\hline$(0.50,1.00)$ & $4,21,1.161,1.345$ & $6,23,1.231,1.182$ \\
\hline$(0.50,1.50)$ & $4,19,1.188,1.275$ & $6,22,1.250,1.145$ \\
\hline$(0.50,2.00)$ & $4,19,1.188,1.275$ & $7,23,1.173,1.311$ \\
\hline
\end{tabular}

TABLE III.: ATS 1 VALUES OF THE VSSI $\bar{X}$ Chart AND Shewhart $\bar{X}$

\begin{tabular}{c|cc|c}
\hline \multicolumn{3}{c}{ CHART } \\
\hline \multirow{4}{*}{} & \multicolumn{3}{c}{ ATS $_{1}$} \\
\cline { 2 - 3 }$\delta$ & VSSI $\bar{X}$ Chart & \multirow{2}{*}{ Shewhart $\bar{X}$ Chart } \\
\cline { 2 - 3 }$\delta$ & $t_{1}=0.10$ & $t_{1}=0.25$ & 227.72 \\
0.20 & 181.20 & 182.03 & 93.04 \\
0.40 & 27.24 & 27.79 & 18.79 \\
1.80 & 3.80 & 4.26 & 5.61 \\
1.60 & 1.51 & 1.89 & 2.44 \\
2.00 & 1.09 & 1.31 & 1.47 \\
\hline \multirow{4}{*}{$\delta$} & 1.02 & 1.15 & Shewhart $\bar{X}$ Chart \\
\cline { 2 - 3 }$\delta$ & \multicolumn{3}{c}{$n_{0}=5$} \\
\cline { 2 - 3 } 0.20 & $t_{1}=0.10$ & $t_{1}=0.25$ & 177.73 \\
0.40 & 122.96 & 124.43 & 56.59 \\
& 13.07 & 13.72 & \\
\hline
\end{tabular}




\begin{tabular}{c|cc|c}
\multirow{2}{*}{0.80} & 2.02 & 2.47 & 8.86 \\
1.20 & 1.09 & 1.35 & 2.66 \\
1.60 & 1.01 & 1.10 & 1.39 \\
2.00 & 1.00 & 1.02 & 1.08 \\
\hline \multirow{3}{*}{$\delta$} & \multicolumn{3}{|c}{$n_{0}=7$} \\
\cline { 2 - 3 } & \multicolumn{3}{|c}{ VSSI $\bar{X}$ Chart } \\
\cline { 2 - 3 } & $t_{1}=0.10$ & $t_{1}=0.25$ & Shewhart $\bar{X}$ Chart \\
\hline 0.20 & 91.77 & 92.97 & 120.86 \\
0.40 & 8.54 & 8.97 & 29.95 \\
0.80 & 1.61 & 1.83 & 6.11 \\
1.20 & 1.08 & 1.18 & 2.24 \\
1.60 & 1.01 & 1.03 & 1.19 \\
2.00 & 1.00 & 1.00 & 1.08 \\
\hline \multirow{4}{*}{$\delta$} & \multicolumn{3}{|c}{$n_{0}=10$} \\
\cline { 2 - 3 }$\delta$ & $t_{1}=0.10$ & $t_{1}=0.25$ & Shewhart $\bar{X}$ Chart \\
\cline { 2 - 3 } 0.20 & 65.03 & 66.57 & 94.52 \\
0.40 & 5.42 & 5.89 & 20.26 \\
0.80 & 1.27 & 1.44 & 4.37 \\
1.20 & 1.03 & 1.07 & 1.87 \\
1.60 & 1.00 & 1.01 & 1.07 \\
2.00 & 1.00 & 1.00 & 1.01 \\
\hline
\end{tabular}

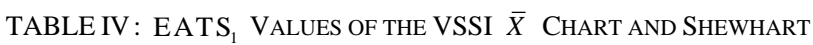

\begin{tabular}{|c|c|c|c|}
\hline \multicolumn{3}{|c|}{ EATS $_{1}$} & \\
\hline \multirow[b]{3}{*}{$\left(\delta_{\min }, \delta_{\max }\right)$} & \multicolumn{3}{|c|}{$n_{0}=3$} \\
\hline & \multicolumn{2}{|c|}{ VSSI $\bar{X}$ Chart } & \multirow[b]{2}{*}{ Shewhart $\bar{X}$ Chart } \\
\hline & $t_{1}=0.10$ & $t_{1}=0.25$ & \\
\hline$(0.30,1.00)$ & 14.54 & 14.71 & 46.77 \\
\hline$(0.30,1.50)$ & 9.96 & 10.11 & 29.53 \\
\hline$(0.30,2.00)$ & 7.67 & 7.81 & 21.44 \\
\hline$(0.50,1.00)$ & 6.25 & 6.46 & 26.52 \\
\hline$(0.50,1.50)$ & 4.37 & 4.55 & 15.96 \\
\hline$(0.50,2.00)$ & 3.45 & 3.62 & 11.31 \\
\hline \multirow[b]{3}{*}{$\left(\delta_{\min }, \delta_{\max }\right)$} & \multicolumn{3}{|c|}{$n_{0}=5$} \\
\hline & \multicolumn{2}{|c|}{ VSSI $\bar{X}$ Chart } & \multirow{2}{*}{ Shewhart $\bar{X}$ Chart } \\
\hline & $t_{1}=0.10$ & $t_{1}=0.25$ & \\
\hline$(0.30,1.00)$ & 7.75 & 7.95 & 26.60 \\
\hline$(0.30,1.50)$ & 5.30 & 5.46 & 16.59 \\
\hline$(0.30,2.00)$ & 4.12 & 4.26 & 12.08 \\
\hline$(0.50,1.00)$ & 3.29 & 3.51 & 13.30 \\
\hline$(0.50,1.50)$ & 2.39 & 2.57 & 7.94 \\
\hline$(0.50,2.00)$ & 1.97 & 2.10 & 5.71 \\
\hline \multirow[b]{3}{*}{$\left(\delta_{\min }, \delta_{\max }\right)$} & \multicolumn{3}{|c|}{$n_{0}=7$} \\
\hline & \multicolumn{2}{|c|}{ VSSI $\bar{X}$ Chart } & \multirow{2}{*}{ Shewhart $\bar{X}$ Chart } \\
\hline & $t_{1}=0.10$ & $t_{1}=0.25$ & \\
\hline$(0.30,1.00)$ & 5.05 & 5.28 & 15.84 \\
\hline$(0.30,1.50)$ & 3.53 & 3.71 & 11.13 \\
\hline$(0.30,2.00)$ & 2.82 & 2.96 & 8.88 \\
\hline$(0.50,1.00)$ & 2.25 & 2.46 & 6.89 \\
\hline$(0.50,1.50)$ & 1.72 & 1.87 & 4.67 \\
\hline$(0.50,2.00)$ & 1.49 & 1.60 & 3.96 \\
\hline \multirow[b]{3}{*}{$\left(\delta_{\min }, \delta_{\max }\right)$} & \multicolumn{3}{|c|}{$n_{0}=10$} \\
\hline & \multicolumn{2}{|c|}{ VSSI $\bar{X}$ Chart } & \multirow[b]{2}{*}{ Shewhart $\bar{X}$ Chart } \\
\hline & $t_{1}=0.10$ & $t_{1}=0.25$ & \\
\hline$(0.30,1.00)$ & 3.28 & 3.52 & 10.56 \\
\hline$(0.30,1.50)$ & 2.40 & 2.57 & 7.71 \\
\hline$(0.30,2.00)$ & 2.00 & 2.12 & 6.04 \\
\hline$(0.50,1.00)$ & 1.61 & 1.79 & 4.94 \\
\hline$(0.50,1.50)$ & 1.33 & 1.46 & 3.85 \\
\hline$(0.50,2.00)$ & 1.23 & 1.31 & 2.83 \\
\hline
\end{tabular}

\section{CONCLUSION}

In this paper, the performance of the VSSI $\bar{X}$ chart is evaluated in terms of the EATS criterion. The optimal parameters are computed to minimize the $\mathrm{EATS}_{1}$ values for all considered process shift ranges. No attempt has been made in the current literature; hence, it could be viewed as a useful suggestion, and especially in real-life situations that practitioners rarely know the exact process shift sizes. The VSSI $\bar{X}$ chart [17] has been successfully proved that it is an effective chart for monitoring the process mean, in terms of ATS criterion. The results indicate that the VSSI $\bar{X}$ chart is superior to the Shewhart $\bar{X}$ chart, in terms of ATS and EATS criteria. The VSSI $\bar{X}$ chart can be superimposed on the Shewhart $\bar{X}$ chart, this means that the computational difficulty between the VSSI $\bar{X}$ and Shewhart $\bar{X}$ charts is not much different. Therefore, it should be given higher consideration than the Shewhart $\bar{X}$ chart. Additionally, the flexibility in varying the sample size and sampling interval is able to reduce the cost of the process and at the same time is able to detect an out-of-control signal quicker. Due to certain constraints in real-life situations, practitioners are able to change the VSSI $\bar{X}$ chart to the VSI $\bar{X}$ or VSS $\bar{X}$ charts, by fixing the sampling interval or sample size, respectively. Thus, the practitioners can select his preferred optimal chart to monitor the process mean for their desired situations. In future research, the evaluation of the EATS performance can be extended to other advanced control charts, such as run sum, exponentially weighted moving average (EWMA) and cumulative sum (CUSUM) control charts.

\section{ACKNOWLEDGMENT}

Special thanks to School of Management, Universiti Sains Malaysia, Malaysia.

\section{REFERENCES}

[1] K. W. Khaw, X. Y. Chew, W. C. Yeong, and S. L. Lim, "Optimal design of the synthetic control chart for monitoring the multivariate coefficient of variation," Chemometrics and Intelligent Laboratory Systems, vol. 186, no. 1, pp. 33-40, 2019.

[2] X. L. Hu, P. Castagliola, and A. A. Tang, "Conditional design of the CUSUM median chart for the process position when process parameters are unknown," Journal of Statistical Computation and Simulation, vol. 89, no. 13, pp. 2468-2488, 2019.

[3] X. Y. Chew, K. W. Khaw, and W. C. Yeong, "The efficiency of run rules schemes for the multivariate coefficient of variation: A Markov chain approach," Journal of Applied Statistics, 2019.

[4] A. Haq, and M. B. C. Khoo, "Memory-type multivariate control charts with auxiliary information for the process mean," Quality and Reliability Engineering International, vol. 35, no. 1, pp. 192-203, 2019.

[5] M. Riaz and F. Muhammad, "An application of control charts in manufacturing industry," Journal of Statistical and Econometric Methods, vol. 1, no. 1, pp. 77-92, 2012.

[6] R. Walter, M. Higgins, and H. Roth, "Applications of control charts," The CPA Journal, pp. 90-93, 95, April 1990.

[7] B. A. Steward, R. E. James, K. F. Knowlton, M. L. McGilliard, and M. D. Hanigan, "An example of application of the process control charts to feed management on dairy farms," The Professional Animal Scientis, vol. 27, no. 6, pp. 571-573, 2011.

[8] X. Y. Chew, M. B. C. Khoo, K. W. Khaw, W. C. Yeong, and Z. L. Chong, "A proposed variable parameter control chart for monitoring the multivariate coefficient of variation," Quality and Reliability Engineering International, 2019.

[9] A. F. B. Costa and M. S. De Magalhaes, "An adaptive chart for monitoring the process mean and variance," Quality and Reliability Engineering International, vol. 23, no. 7, pp. 821-831, 2007.

[10] S. Psarakis, "Adaptive control charts: Recent developments and extensions," Quality and Reliability Engineering International, vol. 31, no. 7, pp. 1265-1280, 2015. 
[11] M. S. De Magalhaes and F. D. M. Neto, "Joint economic model for totally adaptive $\bar{X}$ and $R$ charts," European Journal of Operational Research, vol. 161, no. 1, pp. 148-161, 2005.

[12] E. K. Epprecht, A. F. B. Costa, and F. C. T. Mendes, "Adaptive control charts for attributes," IIE Transactions, vol. 35, no. 6, pp. 567$582,2003$.

[13] W. A. Jensen, G. R. Bryce, M. R. Reynolds Jr, "Design issues for adaptive control charts," Quality and Reliability Engineering International, vol. 24, no. 4, pp. 429-445, 2008.

[14] M. R. Reynolds Jr, R. W. Amin, and J. C. Nachlas, “ $\bar{X}$ charts with variable sampling interval,” Technometrics, vol. 30, no. 2, pp. 181192, 1988.

[15] S. S. Prabhu, G. C. Runger, and J. B. Keats, “ $\bar{X}$ with adaptive sample sizes," International Journal of Production Research, vol. 31 , no. 12 , pp. 2893-2909, 1993.

[16] A. F. B. Costa, " $\bar{X}$ charts with variable sample size," Journal of Quality Technology, vol. 26, no. 3, pp. 155-163, 1994.

[17] S. S. Prabhu, D. C. Montgomery, and G. C. Runger, "A combined adaptive sample size and sampling interval $\bar{X}$ control scheme," Journal of Quality Technology, vol. 26, no.3, pp. 164-176, 1994.

[18] A. F. B. Costa, " $\bar{X}$ charts with variable parameters," Journal of Quality Technology, vol. 31, no. 4, pp. 408-416, 1999.

[19] C. C. Yang, and S. F. Yang, "Optimal variable sample size and sampling interval 'mean squared error' chart," The Service Industries Journal, vol. 33, no. 6, pp. 652-665, 2013.

[20] S. B. Mahadik, and D. T. Shirke, "A special variable sample size and sampling interval $\bar{X}$ chart," Communications in Statistics - Theory and Methods, vol. 38, no. 8, pp. 1284-1299, 2009.

[21] S. L. Lim, M. B. C. Khoo, W. L. Teoh, and M. Xie, "Optimal designs of the variable sample size and sampling interval $\bar{X}$ chart when process parameters are estimated," International Journal of Production Economics, vol. 166, no. 1, pp. 20-35, 2015.

[22] Z. Wu, Y. Tian, and S. Zhang, "Adjusted-loss-function charts with variable sample sizes and sampling intervals, Journal of Applied Statistics, vol. 32, no. 3, pp. 221-242, 2005.

[23] X. Cheng and F. Wang, "VSSI median control chart with estimated parameters and measurement errors," Quality and Reliability Engineering International, vol. 34, no. 5, pp. 867-881, 2018.

[24] H. Sabahno and A. Amiri, "The effect of measurement errors on the performance of variable sample size and sampling interval control chart," International Journal of Engineering Transactions A: Basics, vol. 30, no. 7, pp. 995-1004, 2017.

[25] S. Saha, M. B. C. Khoo, M. H. Lee, and A. Haq, "A variable sample size and sampling interval control chart for monitoring the process mean using auxiliary information," Quality Technology \& Quantitative Management, vol. 16, no. 4, pp. 389-406, 2019.

[26] K. W. Khaw, M. B. C. Khoo, W. C. Yeong, and Z. Wu, "Monitoring the coefficient of variation using a variable sample size and sampling interval control chart," Communications in Statistics - Simulation and Computation, vol. 46, no. 7, pp. 5772-5794, 2017.

[27] Z. T. Kosztyan, and A. I. Katona, "Risk-based X-bar chart with variable sample size and sampling interval," Computers \& Industrial Engineering, vol. 120, no. 1, pp. 308-319, 2018.

[28] S. B. Mahadik, and D. T. Shirke, "A special variable sample size and sampling interval Hotelling's $T^{2}$ chart," The International Journal of Advanced Manufacturing Technology, vol. 53, no. 1-4, pp. 379-384, 2011.

[29] K. W. Khaw, M. B. C. Khoo, P. Castagliola, and M. A. Rahim, "New adaptive control charts for monitoring the multivariate coefficient of variation," Computers \& Industrial Engineering, vol. 126, no. 1, pp. 595-610, 2018
[30] Y. K. Chen, "Economic design of $T^{2}$ control charts with the VSSI sampling scheme," Quality \& Quantity, vol. 43, no. 1, pp. 109-122, 2009.

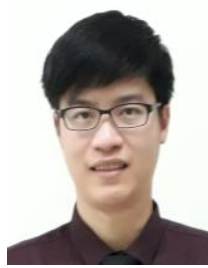

K. W. Khaw is a senior lecturer in the School of Management, Universiti Sains Malaysia (USM). He received his $\mathrm{Ph} . \mathrm{D}$. in statistical quality control from USM. He has featured in prominent international publications, such as Chemometrics and Intelligent Laboratory System, Computers \& Industrial Engineering, Quality and Reliability Engineering International. His research interests are in statistical quality control, advanced analytics, and operations research. His efforts and excellence have been acknowledged and awarded at several dignified platforms.

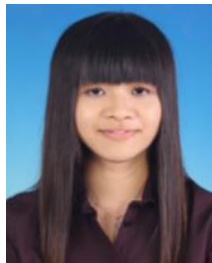

X. Y. Chew is a senior lecturer in the School of Computer Sciences, Universiti Sains Malaysia (USM). She received her Ph.D. in statistical quality control from USM. Her research papers are published in several renowned international peer-reviewed journals, such as Computers \& Industrial Engineering, Quality and Reliability Engineering International and etc. Her research areas are in statistical quality control, robust statistics, data/text mining, machine learning, natural language processing, artificial intelligence and operations research.

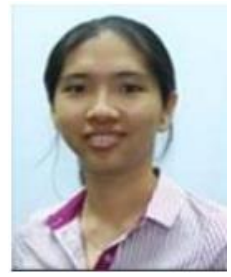

S. Y. Teh is a senior lecturer in School of Management, Universiti Sains Malaysia (USM). She obtained her $\mathrm{Ph} . \mathrm{D}$. in statistical quality control from USM. She was a recipient of the meritorious International Congress of Mathematicians 2014 Travel Fellowship Fund to Seoul Korea awarded by the International Mathematical Union. She was also recipient of Outstanding Dissertation Award 2014 awarded by Industrial Engineering and Operations Management Society. She was also a recipient for the Malaysian Mathematical Sciences Society Award 2013 for Ph.D. thesis. Her research papers are published in several renowned international peer-reviewed journals. Her research interests are in statistical quality control, robust statistics, data mining, operations management, and quality management.

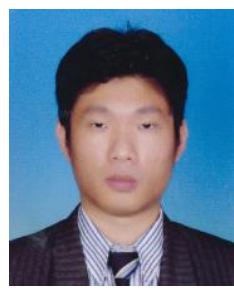

W. C. Yeong is an associate professor in the School of Mathematical Sciences, Sunway University, Malaysia. He obtained his Ph.D. in statistical quality control from USM. He was a recipient of the Certificate of Outstanding Contribution in Reviewing awarded by Elsevier in 2017. He was also awarded Malaysian Mathematical Sciences Society Award 2015 for Ph.D. thesis. He specializes in statistical quality control. $\mathrm{He}$ publishes extensively in international journals. 